\title{
Visual and mechanical control of postural and kinetic tremor in cerebellar system disorders
}

\author{
JEROME N SANES,${ }^{*}$ PETER A LEWITT $\dagger$ KARL-HEINZ MAURITZ \\ From the Human Motor Control Section, Medical Neurology Branch, National Institute of Neurological and \\ Communicative Disorders and Stroke, National Institutes of Health, Bethesda, ${ }^{*}$ Lafayette Clinic, Detroit, \\ USA† and Rehabilitationszentrum, der Universität zu Köln, Federal Republic of Germany $\ddagger$
}

SUMMARY The influence of alterations in visual guidance and somaesthetic sensory inputs was studied in five patients with kinetic and postural tremor characteristic of cerebellar impairment. The patients performed wrist flexion-extension movements or movements about the shoulder with or without visual guidance. Different types of mechanical loads were applied to oppose the wrist movements. The tremor was greatest when the patients used visual cues to guide movements. Kinetic tremor was substantially suppressed during performance of similar movements that were not guided directly by vision. Viscous loads suppressed the tremor nearly linearly, whereas constant loads opposing extension enhanced the tremor. The postural tremor was not observed during isometric contractions. These results support the view that processing of visual information contributes to the impairment of movement in disorders with cerebellar-type tremor and that certain somaesthetic inputs can selectively influence the generation of postural tremor.

The cerebellum receives an abundant influx of somatic sensory and visual information. ${ }^{1-3}$ Although, cerebellar dysfunction is not typically accompanied by impairments in basic discrimination tests, ${ }^{45}$ deficits due to cerebellar disease are generally evident when visual and somaesthetic sensory stimuli need to be integrated into involuntary or voluntary movements. For example, long-latency stretch reflexes are enhanced in certain types of cerebellar deficits, ${ }^{6}$ and arm and eye step and pursuit movements are abnormal in cerebellar dysfunction. ${ }^{7-11}$ These results, and additional work on animals and humans, support a prevalent view that the cerebellar system contributes to integration of sensory information before and during the performance of movements. ${ }^{12}$

One issue of continuing interest with respect to cerebellar dysfunction is the contribution of visual and somaesthetic inputs to the production of cerebellar postural and kinetic tremor. Damage at a number of sites in the cerebellum or its outflow pathways can produce characteristic patterns of tremor. One early

Address for reprint requests: Dr Jerome N Sanes, NINCDS, Building 10, Room 5N-226, Bethesda, MD 20892, USA.

Received 13 October 1987 and in revised form 23 February 1988. Accepted 7 March 1988 view $^{41314}$ for the origin of tremor is that the damage to cerebellar circuits permits a series of overcorrections that develop into oscillatory errors in performance. While some experimental evidence supports this concept of a primarily central origin of kinetic and postural tremor, ${ }^{15-18}$ other evidence is inconsistent with this model. With experimentally induced cerebellar dysfunction in non-human primates, the external mechanical conditions affecting a limb have been shown to influence the production of cerebellar kinetic and postural tremor. ${ }^{19}$ Additionally, and in contrast to the early view, ${ }^{41314}$ it has been reported that alterations in visual guidance do not affect the magnitude of cerebellar postural tremor. ${ }^{20}$ However, it appears that manipulations of the visual environment influence the amount of dysmetria in movements performed by patients with cerebellar ataxia. $^{7}$

The present study investigated the influence of visual guidance on human cerebellar-type tremor and examined the effect of various types of mechanical loading on cerebellar postural and kinetic tremor. In addition to demonstrating major influences of sensory inputs on the production of cerebellar tremor, these observations offer additional practical insights into rehabilitative strategies that can be offered to patients disabled with cerebellar-type tremor. 


\section{Materials and methods}

Five patients with postural and kinetic tremor were studied. In each case, the tremor was characterised by rhythmic, 3 to $5 \mathrm{~Hz}$, to and fro movements in an upper extremity. The neurological diagnosis of the patients included structural and degenerative disease (table). The patient with a red nucleus tumour contralateral to the limbs with tremor, though without a cerebellar lesion, had typical clinical features found in cerebellar outflow disorders and was studied for comparison.

\section{Accelerometry}

Patients were seated and a triaxial accelerometer (Wilcoxon Research Inc., Model 139) was securely strapped to the dorsal surface of the right hand between the metacarpophalangeal and wrist joints. The accelerometer had three piezoelectric strips mounted orthogonally within protective shields to detect motion in the $x$ (lateral), $y$ (vertical), and $z$ (sagittal) planes. The complete apparatus weighed $22.5 \mathrm{~g}$. The sensitivity of the piezoelectric elements was approximately $60 \mathrm{mV} / \mathrm{g}$. The voltages proportional to displacement of the piezoelectric elements were amplified with a band pass frequency of 2 to $40 \mathrm{~Hz}$. The amplifier rolloff was $6 \mathrm{~dB}$ per octave below $2 \mathrm{~Hz}$ and $18 \mathrm{~dB}$ per octave above $40 \mathrm{~Hz}$. After amplification and filtering, data from each axis of movement were led to separate channels of a 10-bit A/D converter of a PDP 11/03 minicomputer and sampled at $100 \mathrm{~Hz}$.

Involuntary movement was evaluated for three activated movements or postures of the arm. These movements were (1) an intended constant posture at $90^{\circ}$ of horizontal arm flexion and $90^{\circ}$ of forward elevation of the arm, (2) a repetitive vertical movement in the sagittal plane from shoulder level to the knee, and (3) a repetitive horizontal movement with an excursion of the approximate distance between the shoulders performed at a level slightly below the shoulder. For all tasks the elbow was extended fully. Patients were allowed to choose a desired speed; they typically performed about one movement cycle per second. When patients performed under the visually targeted condition, the end-point of the movements or the target for postural maintenance was provided by the outstretched hands of the examiner. For

Table Patient description

\begin{tabular}{|c|c|c|c|}
\hline $\begin{array}{l}\text { Patient } \\
\text { No. }\end{array}$ & Sex & $\begin{array}{l}\text { Age } \\
(y r)\end{array}$ & Diagnosis \\
\hline $\mathrm{C} 01^{*}$ & Male & 58 & $\begin{array}{l}\text { Unilateral cerebellar infarct } \\
\text { in the region of the } \\
\text { dentate nucleus and } \\
\text { superior cerebellar } \\
\text { peduncle of the } \\
\text { nondominant side }\end{array}$ \\
\hline $\mathrm{CO} 2^{*}$ & Female & 65 & $\begin{array}{c}\text { Olivopontocerebellar } \\
\text { degeneration }\end{array}$ \\
\hline $\mathrm{CO3t}$ & Female & 35 & $\begin{array}{l}\text { Multiple sclerosis, cerebellar } \\
\text { and brainstem lesions }\end{array}$ \\
\hline $\mathrm{C04}{ }^{*}$ & Female & 70 & $\begin{array}{l}\text { Vascular tumour in the } \\
\text { region of the red nucleus, } \\
\text { nondominant side }\end{array}$ \\
\hline $\mathrm{C} 23_{+}^{+}$ & Male & 55 & $\begin{array}{l}\text { Olivopontocerebellar } \\
\text { degeneration }\end{array}$ \\
\hline
\end{tabular}

* Examined in accelerometry and torque motor studies.

+ Examined only with accelerometry studies.

$\ddagger$ Examined only with torque motor studies. each posture or movement, three 20 second samples of movement were digitised. The postures and movements were performed with and without visual control. For trials without visual control, patients were asked either to close their eyes or look elsewhere and to execute movements similar to the size of those that were performed just before with a visual target.

Data were analysed offline with a PDP-11 computer. Acceleration data from each of the $x, y$, and $z$ axes were first analysed independently and then combined, after digital filtering and integration, to derive a statistic of the total distance travelled in each 20 s test epoch. For each axis, the RMS value of acceleration was derived; this value was then used for integration and summation across the three axes to derive the total distance travelled. A spectral analysis was done with standard fast-Fourier analysis procedures and the proportion and absolute values of power in $0.5 \mathrm{~Hz}$ band widths between 1.5 and $25 \mathrm{~Hz}$ were derived.

\section{Torque motor studies}

Patients were seated and the forearm was stabilised between two padded plates. The extended hand was placed between two padded plates of a handle that was attached to the axle of a low-friction, brushless DC torque motor (Aeroflex TQ64). The hand was hidden from the patient's view, and the wrist was positioned directly over the axle of the torque motor. The patient viewed a visual display that showed a position and a target cursor. The location of the target cursor was computer controlled, and the position cursor corresponded to the location of the hand as controlled by rotation of the torque motor axle. Patients were instructed to orient the torque motor handle so that the position cursor and the target cursor were aligned. The size of the target cursor was varied from $6^{\circ}$ to $20^{\circ}$ to accommodate the tremor amplitude of each patient. The cursor alignments had to be maintained for 1.5 to $2.5 \mathrm{~s}$, whereupon the target jumped to a new location. Patients were instructed then to move the hand at their own pace to reestablish the alignment between the cursors. The required movement size was $30^{\circ}$ with an optimal start position of $15^{\circ}$ of extension and a final position of $15^{\circ}$ of flexion. A series of 25 movements was performed for each sensory condition (see below). The handle position was digitised at $200 \mathrm{~Hz}$. Muscle activity was recorded with surface electrodes from the forearm flexor and extensor muscle groups. The signals were amplified with high input impedance AC-coupled devices (Grass Instrument Co. or Bak Electronics, filter settings at $30 \mathrm{~Hz}$ and $3 \mathrm{KHz}$ ) and then full-wave rectified and low pass filtered (4-pole Bessel filter design, $-3 \mathrm{~dB}$ at $50 \mathrm{~Hz}$ ) and then digitised at $200 \mathrm{~Hz}$.

Data were analysed offline by inspecting each trial and marking, with an electronic cursor, periods of time after the patient had reached a final hand position (fig 1). The root mean square of the velocity record was measured in this period.

Visually guided movements For all series of movements, both the position and the target cursors were initially shown on the visual display. At the end of the alignment period, a brief sound, a double ringing of the computer terminal bell signified the cue to begin movement. On trials in which the target cursor remained visible, the target jumped to a new location on the display screen, thereby giving an additional movement cue to the patients. Simultaneous with the occur- 

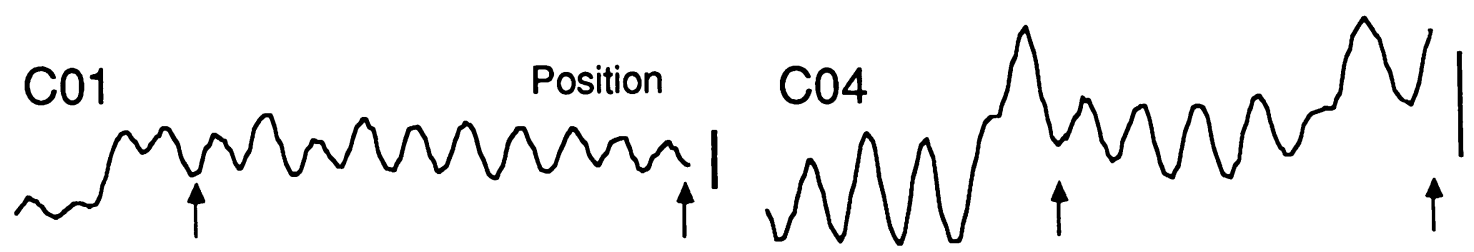

Flexor EMG
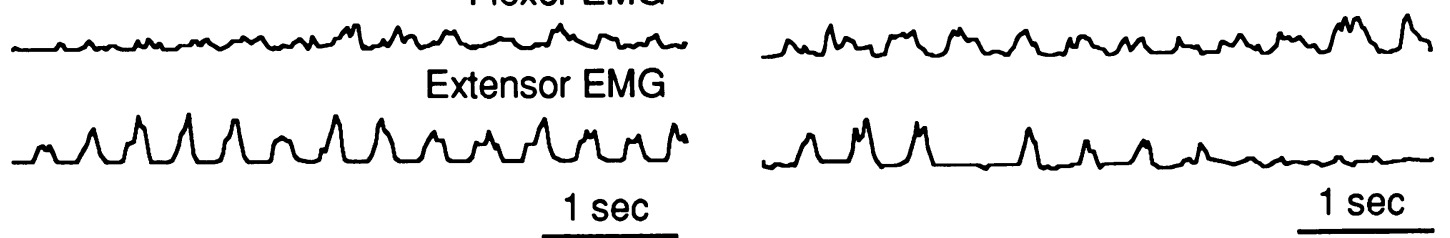

C02
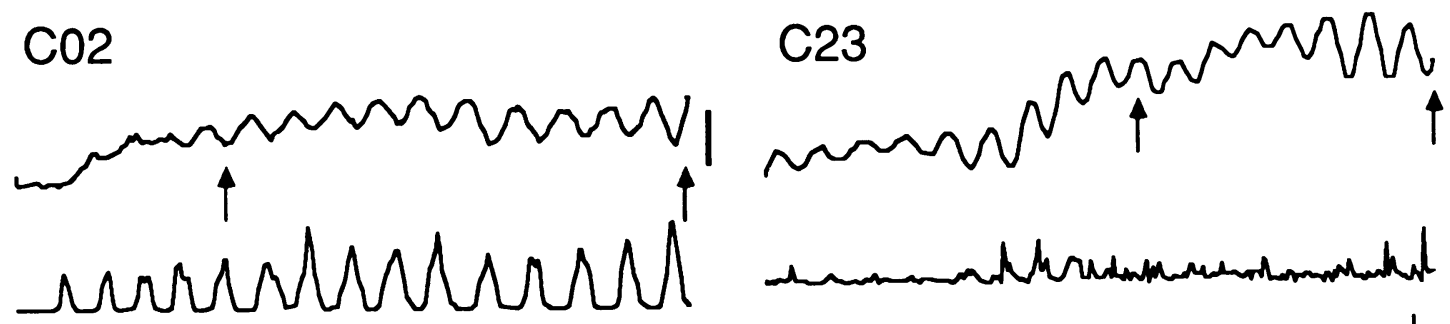

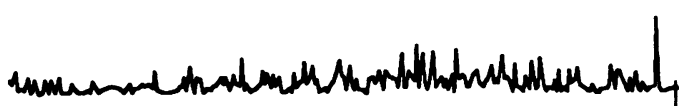

Fig 1 Wrist position and flexor and extensor muscle activity in each of the four patients examined. Kinematic and muscle responses were observed when patients performed voluntary wrist movements toward flexion with the torque motor apparatus. In most instances, tremor in the hand was accompanied by reciprocal bursting in antagonist muscles, although other muscle activity patterns were evident (see C02 and C23). The arrows demarcate the times between which the amount of tremor was calculated. Position calibration $30^{\circ}$ in all records.

rence of the sound a change in the visual display sometimes occurred so as to alter the visual guidance condition. Four such conditions were tested. These were the permutation of both the target and position cursors being present or absent. Each condition (for example, the target cursor present and the position cursor absent) was tested for 25 consecutive trials. Then a new condition was imposed until all four were tested. The complete visual guidance condition (that is, target and position cursors present) was given first, and the order of the remaining conditions varied in a counterbalanced fashion across the subject group.

Mechanical Influences These experiments examined how changes in mechanical loading conditions affected cerebellar postural and kinetic tremor and whether the tremor would be evident when isometric contractions were performed. For all movements, both the target and the position cursors were continuously available for visual guidance. Movements were opposed or assisted by a constant load, a viscous load, or an inertial load. The constant loads were generated by applying a continuous voltage to a control circuit and amplifier system coupled to the torque motor. Constant loads between 0.64
$\mathrm{Nm}$ opposing flexion and $0.64 \mathrm{Nm}$ opposing extension were used. The viscous load was generated by applying negative velocity feedback to the torque motor control circuit. The amount of viscosity was determined by the gain of the negative velocity feedback. Inertial loads were applied by attaching weights of 250,500 , or $1000 \mathrm{~g}$ to the handle of the motor. The resulting moments of inertia were $3.03 \mathrm{~kg} \mathrm{~m}^{2}$ $10^{-3}, 6.05 \mathrm{~kg} \mathrm{~m}^{2} 10^{-3}$ and $12 \cdot 1 \mathrm{~kg} \mathrm{~m}^{2} 10^{-3}$ for the different loads. The patients were not informed as to the type or magnitude of the applied load. A series of 25 trials was presented for each of the viscous, constant, and inertial loads. For isometric contractions about the wrist joint, the hand was inserted between two lightly padded plates of a heavy aluminium frame. The forearm was secured as before. The aluminium frame had strain gauges (in a bridge configuration) that measured deformation of the metal frame caused by wrist flexion or extension. Patients were required to perform separate series of 25 isometric contractions between $4 \mathrm{Nm}$ of flexion to $2 \mathrm{Nm}$ of extension. For loaded movements and isometric contractions, the data were analysed as for visually guided movements. 
Postural maintenance

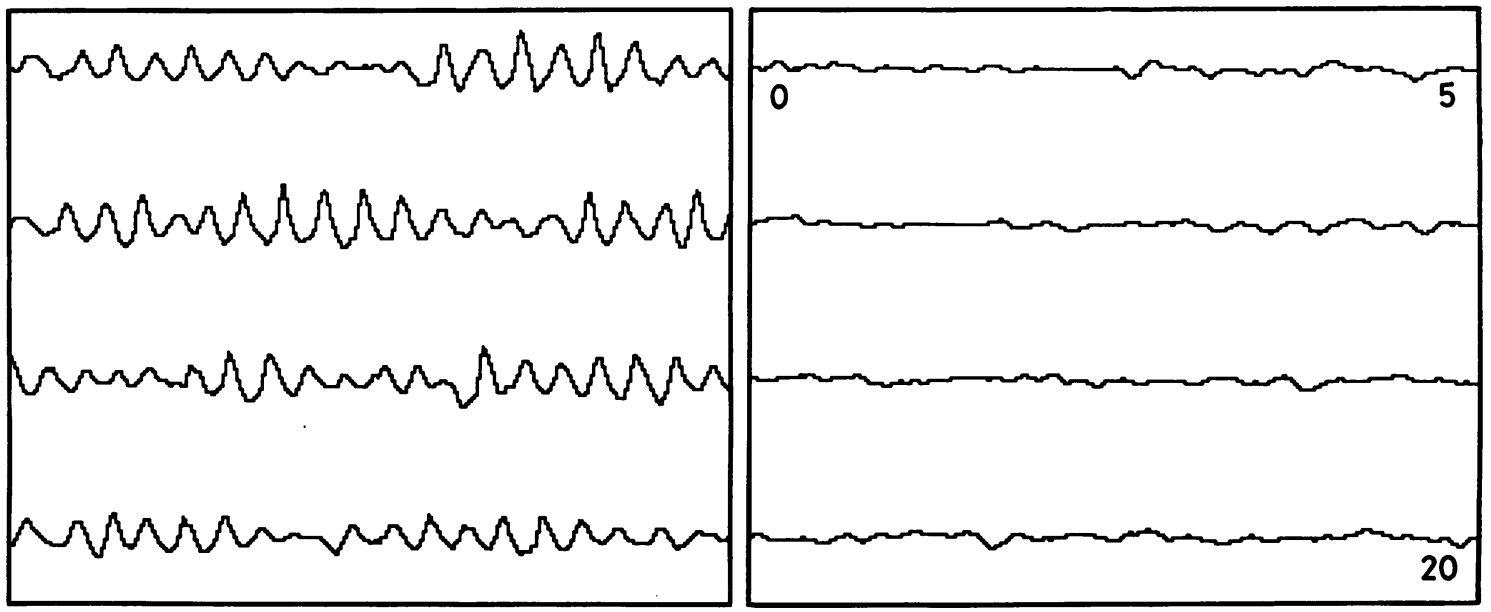

Horizontal movement
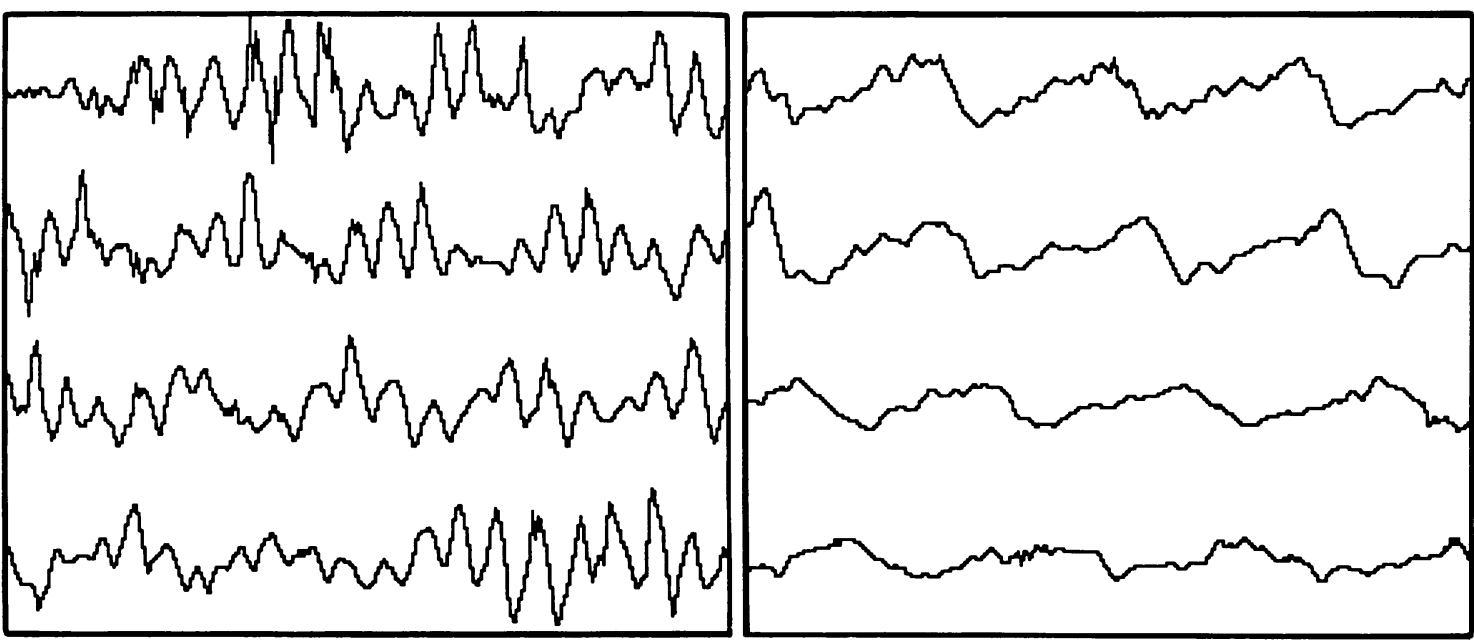

Fig 2 Tremor during postural maintenance and voluntary arm movements. The acceleration records are from patient C03 during intended fixation of the arm and voluntary movements in the horizontal plane. These records are taken from the vertical axis for fixation and from the horizontal axis for the arm movements and are shown during performance with (Targeted) and without (No Target) visual guidance. The numbers in the upper right panel refer to the elapsed time in seconds; each tracing is $5 \mathrm{~s}$ record, in sequence from top to bottom. The frequency of the repetitive voluntary limb movement (about $1 \mathrm{~Hz}$ ) can be seen in the lower right panel. Note the diminution of tremor in the No Target condition.

\section{Results}

Visual guidance

Accelerometry Figure 2 illustrates the acceleration data from one patient during postural maintenance and during horizontal movements performed with and without visual targeting. Postural tremor was greatest when the patient attempted to maintain a constant arm position of $90^{\circ}$ of both horizontal and forward flexion directed at a visual target. During horizontal movements, tremor was nearly always greater when visual targets were present than without external visual targets. For the group of patients, the mean total tremor in the 20 s test was larger during the visually targeted movements or posture than during the untargeted movements or posture $(p<0.05$, fig 3$)$. 


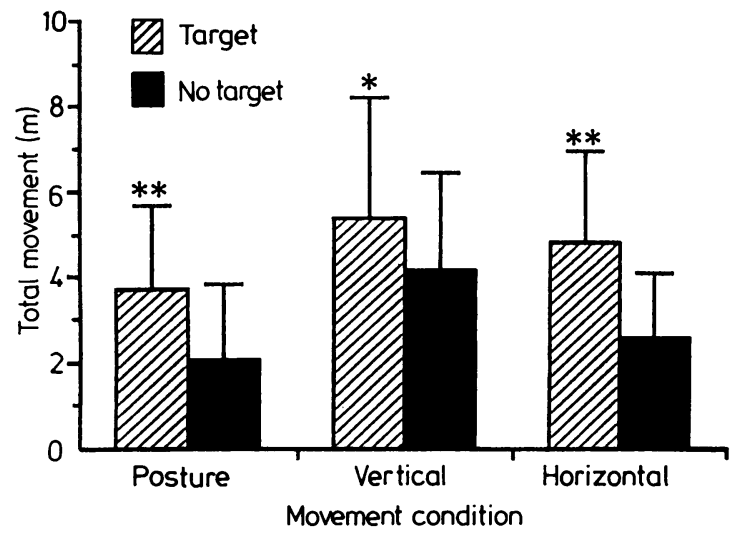

Fig 3 Average tremor expressed as distance travelled. Summary of accelerometry data for postural maintenance and the vertical and horizontal arm movements for multiple 20 s samples. The mean, SEM data for the group of patients are shown. Note that tremor was reduced when visual guidance was eliminated $\left(^{*}=p \leq 0.01,{ }^{* *}=p \leq 0.025\right)$.

The average amount of tremor suppression in the non-visually guided conditions was approximately the same for the three types of movements $(1-2 \mathrm{~m})$, though the relative proportion of suppression was greatest $(60 \%$ to $80 \%)$ for the horizontal movements and the constant arm position. There was no evidence that the frequency of tremor changed in relation to the type of visual targeting.

Torque motors Figure 4 shows the hand position data from one patient during performance of two of the four visual targeting conditions, and fig 5 illustrates the average results from three patients for all visual conditions. Withdrawal of visual guidance, by removing either the target or the position cursor (or both), reduced the amount of tremor. This effect was observed on most trials (see fig 4, bottom). The most effective visual guidance condition for reducing tremor was when the position cursor was absent but the target cursor was seen; all patients tested showed reduction of tremor at the end of movements performed with this type of visual guidance condition. Complete withdrawal of both the position and the target cursors was most effective for reducing tremor in one patient, but ineffective for the other two patients. Removing only the target cursor reduced the amount of tremor for one patient.

\section{Mechanical influences}

Viscous loads Figure 6a illustrates the results from the experiments in which viscous loads of varying intensities opposed wrist movements. The heavier viscous loads suppressed tremor more than the lighter viscous loads. Linear regression analyses yielded correlations between 0.81 and 0.96 relating the amount of viscosity and tremor in individual patients. When considered independently, only one patient (C04) exhibited a significant linear relationship between the magnitude of the viscous load and the amount of tremor $(R=0.96, \mathrm{df}=2, \mathrm{p} \leq 0.05)$. However, pooling the data from all patients revealed a significant linear correlation between the amount of the viscous load and the tremor magnitude $(R=0.83$, $\mathrm{df}=16, \mathrm{p} \leq 0.01)$. Fitting the individual patient data with second-order polynomials resulted in significant correlations ( $p \leq 0.05)$ between the amount of viscosity and the magnitude of postural tremor for all

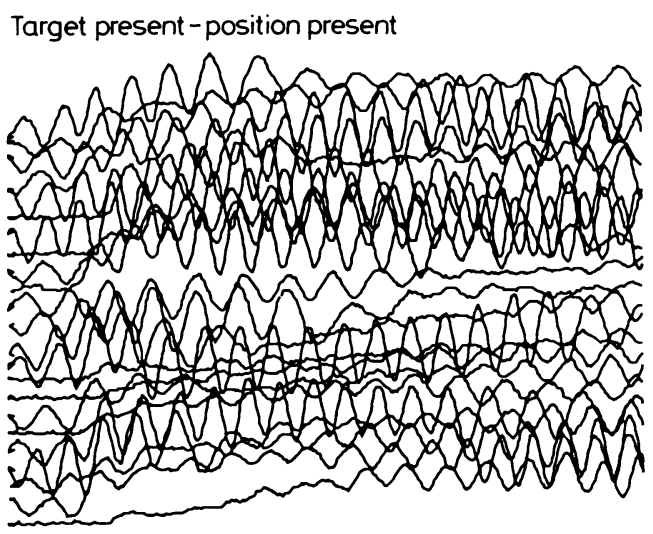

11+1+1+1+1+1+1+1+1+1+1+1+1+1+1+1+1+h

Target present-position absent

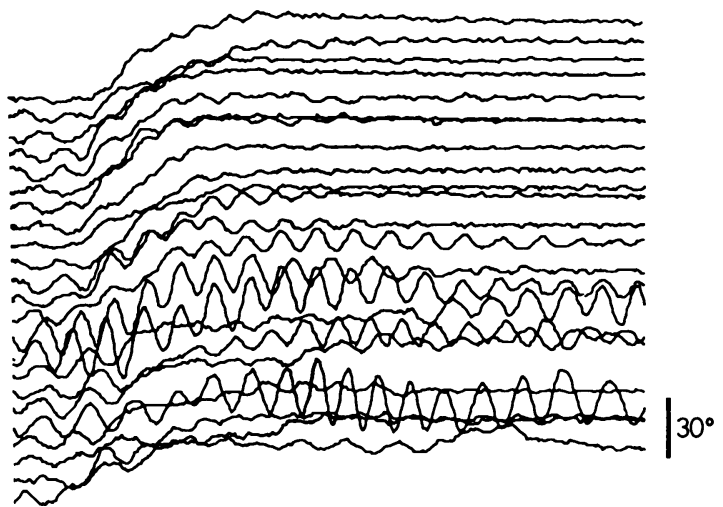

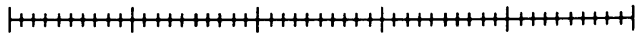

is

Fig 4 Tremor and hand movements. The instantaneous hand position for successive trials performed with (top) or without (bottom) complete visual guidance is shown for patient C02. Note the suppression of tremor when movements were performed when the position cursor was blanked simultaneously with the cue to begin movement. 
patients. The nonlinearity in tremor reduction with respect to the intensity of the viscous load was apparent insofar as movements performed against the lightest viscous load, although of a different magnitude for different patients, resulted in the greatest diminution of tremor. The lightest viscous load reduced tremor by $38 \%$ to $56 \%$ (mean, SEM $=48$, $4.32 \%$ ) compared with the tremor observed during movements performed without viscous resistance. In contrast, the heavier loads reduced tremor only an additional $2 \%$ to $23 \%$ (mean, SEM $=14,2.56 \%$ ). These differences were significant $(t=7 \cdot 615, \mathrm{df}=12$, $\mathrm{p} \leq \mathbf{0 . 0 0 0 1 )}$.

Opposing loads The results concerned with postural tremor when constant loads opposed or assisted wrist flexion are shown in fig $6 \mathrm{~b}$. In general, loads that required patients to activate the wrist extensor muscles in order to maintain an intended position in increased postural tremor in comparison with unloaded movements. For two of the three patients studied with this method, the load that required patients to activate the wrist flexor muscles so as to maintain the intended position decreased the postural tremor. For the remaining patient (C23), although the tremor when flexor loads were opposed seemed greater than during unloaded movements, only the lightest flexor load resulted in enhancement of postural tremor $(p \leq 0.025)$. Additionally, for patient C23 the postural tremor observed when extensor loads were applied was greater than the tremor

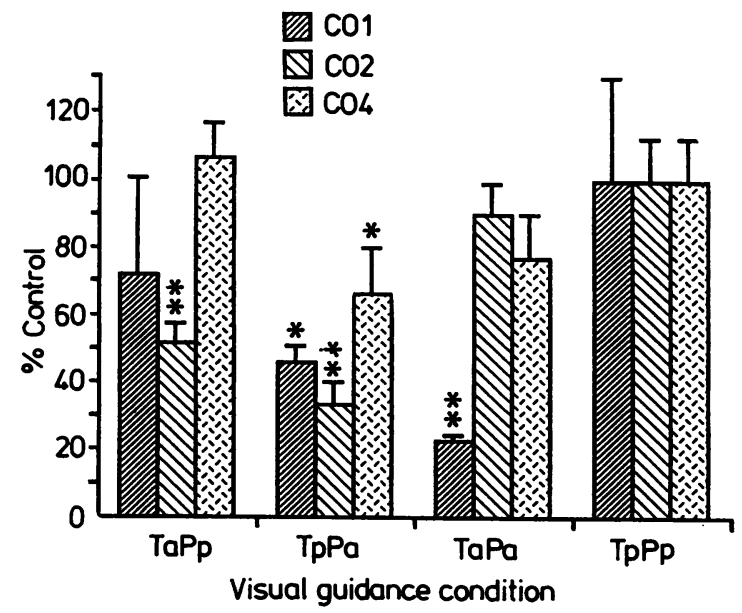

Fig 5 Average tremor for the visual guidance conditions. Summary of the torque motor data. The average data, derived from the RMS of hand velocity, are shown for all patients. $\left(^{*}=p \leq 0.05, * *=p \leq 0.005\right)$. The amount of tremor is expressed relative to tremor magnitude observed in the target cursor present-position cursor present ( $T p P p)$ condition. $T=$ target cursor; $P=$ position cursor, $a=$ absent $; p=$ present . observed when flexor loads were opposed ( $p \leq 0.01$ ). Inertial loads The effect of inertial loads on postural tremor was tested on four patients (fig 6c). For three of the patients, inertial loads of either 500 or $1000 \mathrm{~g}$ applied to the torque motor handle decreased the amount of tremor. The patient with the rubral tumour showed a slight increase in tremor; this was significantly greater than the no-load condition only when the $500 \mathrm{~g}$ load was applied ( $\mathrm{p} \leq 0.05)$. The palliative effects of inertial loads on tremor were not necessarily linear, especially as one patient showed an increase in tremor with an increase of load. Indeed, the tremor of only one patient (C23) was linearly related to the magnitude of the inertial load $(R=$ $0.99, \mathrm{p} \leq 0.01)$; the tremor of the remaining patients showed nonlinear responses when increasing inertial loads opposed movement.

Isometric contractions Figure 7 shows the results from the patient who was separately evaluated during both isometric and isotonic contractions. This patient (C23) exhibited substantial tremor during isotonic contractions when either a flexor or an extensor (not shown) load of $0.64 \mathrm{Nm}$ opposed movement. In contrast, during an isometric contraction that required a step change in torque from 0 to 1 to $4 \mathrm{Nm}$ toward flexion or extension (not shown), there was no evidence of rhythmicity in the torque record. Instead, most trials demonstrated substantial amounts of instability, resembling the qualities of dysmetric movements typically observed in cerebellar disease.

\section{Discussion}

The results of this study indicate that both visual and somaesthetic sensory inputs influence the magnitude of cerebellar kinetic and postural tremor in humans. In particular, patients with cerebellar dysfunction exhibited enhancement of tremor when movements were visually targeted. Additionally, viscous loads, inertial loads, and constant loads opposing flexion all tended to reduce tremor. Finally, there was no evidence of rhythmic involuntary movements when a patient performed voluntary muscle contractions isometrically. This last result indicates that sensory inputs related to muscle stretch and joint rotation may be important in initiating or maintaining tremor in cerebellar patients.

Of particular significance to the interpretation of these data is the nature of postural and kinetic tremor in voluntary movement. Both types of tremor may be present in cerebellar and outflow pathway disorders, as in each of the patients studied, although depending on the particular pathology one or the other pattern may predominate. Each type of tremor needs to be distinguished from the incoordination and decom- 

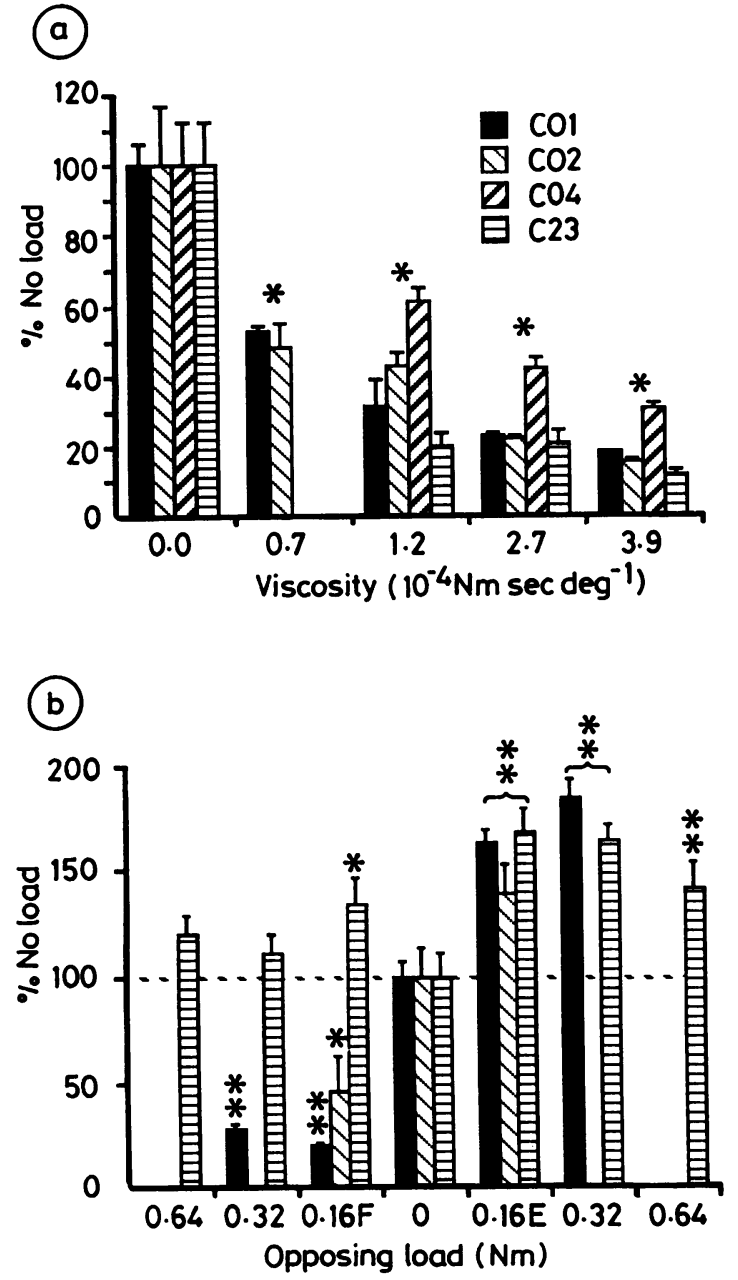

(c)

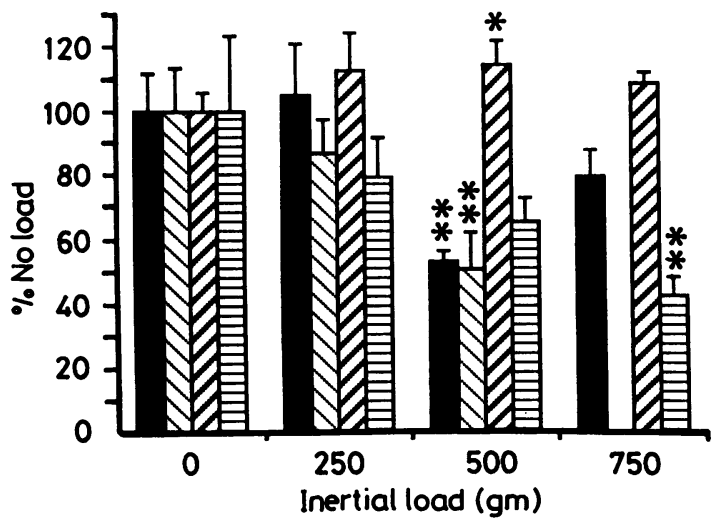

position of movement also found with cerebellar dysfunction. While distinctions have been drawn between the clinical characteristics of postural and kinetic tremors in cerebellar disease, ${ }^{2122}$ our data suggest no significant difference between either type in their sensitivity to manipulation of visual guidance. Hence, it seems possible that postural and kinetic tremors due to cerebellar dysfunction could originate from similar neural pathways.

An interpretation of the dynamics of kinetic tremor in cerebellar disease is that there is a defect in a visually based corrective mechanism that controls the accuracy of voluntary movements. ${ }^{41523}$ This would be similar to recent interpretations of reduced dysmetria in the absence of visual guidance in patients with cerebellar ataxia. ${ }^{7}$ However, this notion has been challenged by the demonstration that the lack of visual guidance during performance of voluntary movement did not alter the amount of kinetic tremor in monkeys with dysfunction of the deep cerebellar nuclei. ${ }^{20}{ }^{24}$ Instead, cerebellar tremor was thought to reflect disorders in short and long-latency reflex pathways. ${ }^{2425}$ For example, a simple alteration in the stretch reflex feedback system, such as latency or gain changes, could cause oscillations in an otherwise stable system. ${ }^{25}$

Several observations from the present data argue against cerebellar kinetic and postural tremor as reflecting only a corrective mechanism. First, although the tremor was suppressed when the visual feedback loop was opened, some tremor remained. Thus, it was apparent that neither postural nor kinetic tremor were initiated as a direct consequence of intent per se, since the tremors can be dramatically reduced, or even abolished, when the same movements are performed voluntarily but without precise visual guidance. Second, during the isometric contraction task, there was no evidence of rhythmic oscillations in the torque records in spite of the fact that these muscle contractions were performed under closed-loop visual control. Instead of tremor, the isometric step contractions were accompanied by a movement disorder akin to serial dysmetria. In its kinematic profile, serial dysmetria is considerably different from tremor. ${ }^{22} \mathrm{~A}$ third rationale for invoking more than corrective mechanisms in the generation of cerebellar tremor is that the mechanical loads affected the magnitude of

Fig 6 Tremor and mechanical loads. In all sections, the tremor is expressed as the RMS of hand velocity and is plotted relative to the magnitude of the $R M S$ velocity when no load opposed movement. a. Viscous loads. The tremor was reduced for all loads ( $p \leq 0.001)$. b. Opposing loads. Tremor magnitude is displayed for both loads opposing flexion (to the left of the 0 opposing load) and loads opposing extension. $\left(^{*}=p \leq 0.05 ;^{* *}=p \leq 0.01\right)$. c. Inertial loads. $\left(^{*}=p \leq 0.05 ;^{* *}=p \leq 0.01\right)$. 


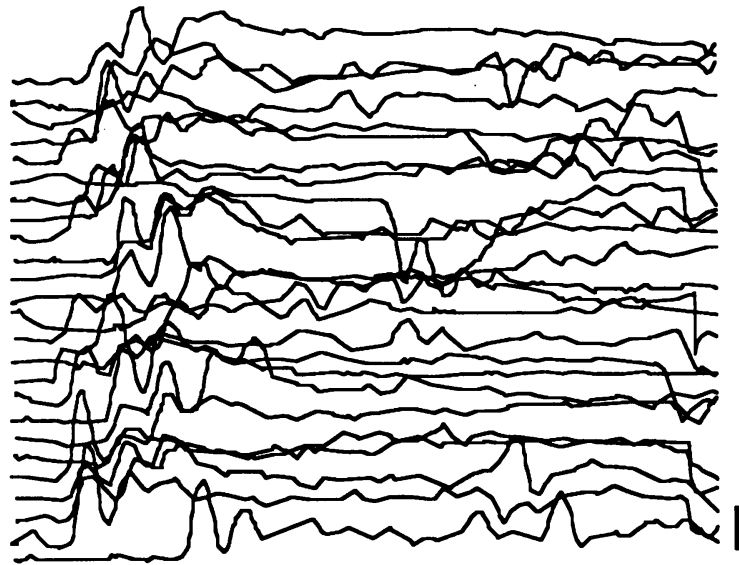

H+H+H+H+H+h

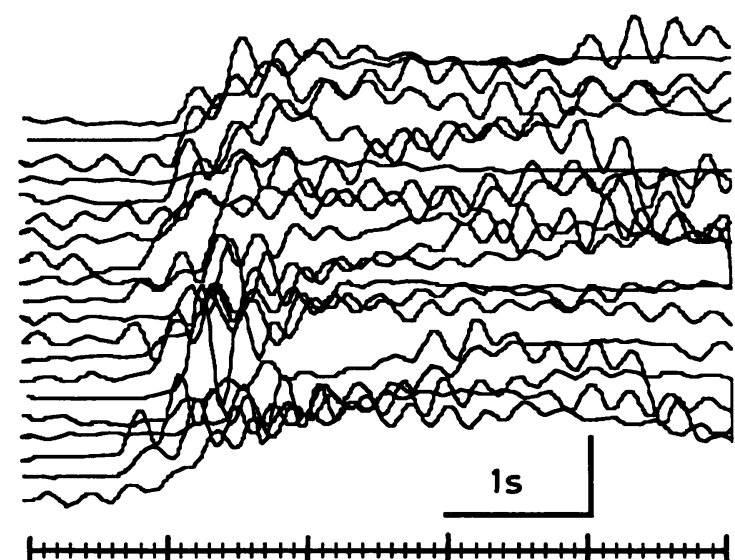

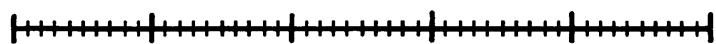

Fig 7 Isometric performance. A series of isometric (left) and isotonic (right) contractions performed separately by patient C23. The constant opposing load was $0.64 \mathrm{Nm}$, and the target isometric torque for the step contraction was $1 \mathrm{Nm}$. Note the absence of rhythmic oscillations in the torque records during the isometric contractions. Calibration for the isometric contractions is $1 \mathrm{Nm}$ and for the isotonic contractions is $30^{\circ}$. The amount of tremor was derived from the RMS of hand velocity (isotonic) or from the RMS first derivative of wrist torque (isometric).

the tremor, sometimes abolishing it, even though the patients performed limb movements with visual guidance. Although some loads might be expected to suppress tremor (for example, viscous loads), it has been shown that some aspects of cerebellar tremor are not susceptible to mechanical loading. ${ }^{2627}$ Finally, the frequency of the postural and kinetic tremor was higher $(2 \cdot 5-4 \mathrm{~Hz})$ than the frequencies $(\sim 2 \cdot 0-2 \cdot 5 \mathrm{~Hz}$, allowing for 200 s minima each in reaction time and correction time) possible to consider that a voluntary corrective mechanism solely generated the disordered movements. Despite, our rejection of the notion that cerebellar tremor is generated only by visually based corrective mechanisms, it was nevertheless clear that visual input had some impact on movement control in patients with cerebellar tremor as it does on cerebellar dysmetria. $^{7}$

The present results fail to support the view that visually based corrective mechanisms are the only cause of tremor, but they also conflict with the results in monkeys showing that withdrawal of visual guidance was ineffective in reducing cerebellar tremor. ${ }^{192024}$ It is likely that differences in methodology can explain the divergent results. The most significant difference may be that in contrast to our patients the monkeys tended not to have tremor when involuntary perturbations or voluntary movements were initiated. Additionally, in an earlier study, ${ }^{19}$ tremor was examined after a mechanical perturbation, so that it is probable that the mechanical characteristics of the limb, rather than voluntary mechanisms, were being measured. In more recent studies, ${ }^{2024}$ even though the voluntary movements were examined in relation to tremor, the movements performed by the monkeys were more rapid than the movements performed by the patients in the current study. Although rapid movements are affected by peripheral inputs, ${ }^{2829}$ it is likely that slower movements are more influenced by visual control. ${ }^{30}$

The influence of constant loads and isometric performance on tremor magnitude may provide some additional insights into the mechanisms of cerebellar postural tremor. In particular, the tremor was selectively enhanced when extensor muscles were activated voluntarily but absent during voluntary isometric contractions. A previous report ${ }^{31}$ demonstrated that finger extension enhanced cerebellar tremor. Thus, it is possible that voluntary activation of extensor muscles contributes to enhancement of postural tremor in patients with cerebellar disease. In view of the absence of tremor during isometric contractions, the contribution of muscle spindle afferents to the maintenance of tremor might be considered. During voluntary ramp displacements of the hand, the discharge pattern of neurons in the cerebellar interpositus deep nucleus was remarkably similar to that of muscle spindle afferents. ${ }^{32}$ In another study, ${ }^{33}$ cellular activity in the interpositus nucleus and muscle spindle activity were correlated to both the hand position and EMG associated with the action tremor of an experimental monkey. The contribution of muscle spindles to cerebellar tremor is likely related to the 
passive length changes of muscles (that is, reflex mechanisms) rather than to mechanisms of alphagamma co-activation. Muscle spindle afferents are activated during both isotonic and isometric voluntary muscle contractions. ${ }^{34} 35$ Therefore the reflexive contributions associated with voluntary movement would seem to be a factor in the appearance of tremor during isotonic contractions and the absence of tremor during isometric performance. The potential muscle spindle, and thus reflex, contribution to cerebellar postural tremor could be supported by observations that stretch reflexes are larger during isotonic contractions than during isometric contractions, even when the initial conditions of torque and position are held constant. ${ }^{3637}$

There are only limited pharmacological options for symptomatic control of cerebellar tremor. ${ }^{38} 39$ However, the contribution of visual input to intention tremor may have important consequences for rehabilitative approaches to the disabilities of cerebellar disease. For example, tremor amplitude may be lessened by directing a patient to execute a goal-directed movement without the use of visual guidance (that is, guiding the movement from memory as to the intended target position). Similarly, physical methods to reduce the severity of kinetic tremor might take into account the ways in which a particular patient's tremor is influenced by mechanical loads. Previous approaches for attenuating tremor by physical means have made use of weights ${ }^{4041}$ or viscous damping devices attached to body appendages. More ideal methods to lessen tremor oscillations can be developed along these principles to apply the particular mechanical load factors observed to decrease tremor.

\section{References}

1 Gilman S, Bloedel JR, Lechtenberg R. Disorders of the Cerebellum, FA Davis Co., 1981

$2 \mathrm{Kim}$ JH, Ebner TJ, Bloedel JR. Comparison of response properties of dorsal and ventral spinocerebellar tract neurons to a physiological stimulus. Brain Res 1986;369:125-35.

3 Glickstein M, May JG, III, Mercier BE. Corticopontine projection in the macaque: the distribution of labelled cortical cells after large injections of horseradish peroxidase in the pontine nuclei. J Comp Neurol 1985;235:343-59.

4 Holmes G. The symptoms of acute cerebellar injuries due to gunshot injuries. Brain 1917;40:461-535.

5 Angel RW. Barognosis in a patient with hemiataxia. Ann Neurol 1980;7:73-7

6 Friedemann H-H, Noth J, Diener HC, Bacher M. Long latency EMG responses in hand and leg muscles: cerebellar disorders. J Neurol Neurosurg Psychiatry 1987;50:71-7

7 Beppu H, Nagaoka M, Tanaka R. Analysis of cerebellar motor disorders by visually guided elbow tracking movement. 2 . Contribution of the visual cues on slow ramp pursuit. Brain 1987;110:1-18.

8 Beppu H, Suda M, Tanaka R. Analysis of cerebellar motor disorders by visually guided elbow tracking movement. Brain 1984;107:787-809.
9 Miall RC, Weir DJ, Stein JF. Visuo-motor tracking during reversible inactivation of the cerebellum. Exp Brain Res 1987:65:455-64.

10 Vilis T, Hore J. A comparison of disorders in saccades and in fast and accurate elbow flexions during cerebellar dysfunction. Prog Brain Res 1986;64:207-15.

11 Optican LM, Robinson DA. Cerebellar-dependent adaptive control of the primate saccadic system. J Neurophysiol 1980;44:1058-76.

12 Brooks VB, Thach WT. Cerebellar control of posture and movement. In: Brooks VB ed. Handbook of Physiology, Section I, the Nervous System, Vol. II, Motor Control, Bethesda, American Physiological Society, 1981: 877-946.

13 Holmes G. Clinical symptoms of cerebellar disease and their interpretation. The Croonian Lectures. II. Lancet 1922: i;1231-7.

14 Holmes G. The cerebellum of man. Brain 1939;62:1-30.

15 Growdon JH, Chambers WW, Liu CN. An experimental study of cerebellar dyskinesia in the rhesus monkey. Brain 1967;90:603-32.

16 Liu CN, Chambers WW. A study of cerebellar dyskinesia in the bilateral deafferented forelimbs of the monkey (Macaca mulatta and Macaca speciosa). Acta Neurobiol Exp Warsaw 1971;31:262-89.

17 Murphey JT, Kwan HC, MacKay WA, Wong YC. Physiological basis of cerebellar dysmetria. Can J Neurol Sci 1975; 38:279-84.

18 Gilman S, Carr D, Hollenberg J. Kinematic effects of deafferentation and cerebellar ablation. Brain 1976;99:311-30.

19 Vilis T, Hore J. Effects of changes in mechanical state of limb on cerebellar intention tremor. J Neurophysiol 1977;40:1214-24.

20 Flament D, Vilis T, Hore J. Dependence of cerebellar tremor on proprioceptive but not visual feedback. Exp Neurol 1984; 84:314-25.

21 Fahn S. Cerebellar tremor: clinical aspects. In: Findley LJ, Capildeo R, eds. Movement Disorders, New York, Oxford University Press, 1984:355-63.

22 Sabra AF, Hallett $M$. Action tremor with alternating activity in antagonist muscles. Neurology 1984;34:151-6.

23 Sutton GG, Sykes K. The effect of withdrawal of visual presentation of errors upon the frequency spectrum of tremor in a manual task. J Physiol (Lond) 1967;190:281-93.

24 Hore J, Flament D. Evidence that a disordered servo-like mechanism contributes to tremor in movements during cerebellar dysfunction. $J$ Neurophysiol 1986;56:123-36.

25 Mauritz KH, Schmitt C, Dichgans J. Delayed and enhanced long latency reflexes as the possible cause of postural tremor in late cerebellar atrophy. Brain 1981;104:97-116.

26 Hömberg V, Hefter H, Reiners K, Freund H-J. Differential effects of changes in mechanical limb properties on physiological and pathological tremor. $J$ Neurol Neurosurg Psychiatry 1987;50:568-79.

27 Lee RG, Stein RB. Resetting of tremor by mechanical perturbations: a comparison of essential tremor and parkinsonian tremor. Ann Neurol 1981;10:523-31.

28 Pëlisson D, Prablanc C, Goodale MA, Jeannerod M. Visual control of reaching movements without vision of the limb. II. Evidence of fast unconscious processes correcting the trajectory of the hand to the final position of a double-step stimulus. Exp Brain Res 1986;62:303-11.

29 Sanes JN. Kinematics and end-point control of arm movements are modified by unexpected changes in viscous loading. $\mathrm{J} \mathrm{Neu}$ rosci 1986;6:3120-7.

30 Woodworth RS. The accuracy of voluntary movements. Psychol Rev Monogr 1899;3:1-114.

31 Chase RA, Cullen JK, Sullivan SA. Modification of intention tremor in man. Nature 1965;206:485-7.

32 Thach WT, Schieber MH, Mink J, Kane S, Horne M. Cerebellar relation to muscle spindles in hand tracking. Prog Brain Res 1986;64:217-24. 
33 Elble RJ, Schieber MH, Thach WT. Activity of muscle spindles, motor cortex and cerebellar nuclei during action tremor. Brain Res 1984;323:330-4.

34 Burke D, Hagbarth, K-E, Skuse NF. Recruitment order of human spindle endings in isometric voluntary contractions. $J$ Physiol (Lond) 1978;285:101-12.

35 Hulliger M, Nordh E, Vallbo ÅB. Direction in muscle spindle afferents related to direction of slow precision movements in man. J Physiol (Lond) 1985;362:437-53.

36 Akazawa K, Milner TE, Stein RB. Modulation of reflex EMG and stiffness in response to stretch of human finger muscle. $J$ Neurophysiol 1983;49:16-27.

37 Kanosue K, Akazawa K, Fujii K. Modulation of reflex activity of motor units in response to stretch of a human finger muscle.
Jap J Physiol 1983;33:995-1009.

38 Legg NJ. Treatment of cerebellar tremor. In: Findley LJ, Capildeo R, eds. Movement Disorders, New York, Oxford University Press, 1984;377-86.

39 Sabra A, Hallett M, Sudarsky L, Mullally W. Treatment of action tremor in multiple sclerosis with isoniazid. Neurology 1982;32:912-13.

40 Hewer RL, Cooper R, Morgan MH. An investigation into the value of treating intention tremor by weighting the affected limb. Brain 1972;95:579-90.

41 Morgan MH, Hewer RL, Cooper R. Application of an objective method of assessing intention tremor-a further study on the use of weights to reduce intention tremor. J Neurol Neurosurg Psychiatry 1975;38:259-64. 